\title{
(-)-Epigallocatechin-3-gallate inhibition of Epstein-Barr virus spontaneous lytic infection involves downregulation of latent membrane protein 1
}

\author{
SUFANG LIU ${ }^{1-3}$, HONGDE $\mathrm{LI}^{1,3}$, MIN TANG ${ }^{1,3}$ and $\mathrm{YACAO}^{1,3}$ \\ ${ }^{1}$ Key Laboratory of Carcinogenesis and Cancer Invasion, Chinese Ministry of Education, Xiangya Hospital, \\ Central South University, Changsha, Hunan 410078; ${ }^{2}$ Division of Hematology, Institute of Molecular Hematology, \\ The Second Xiangya Hospital, Central South University, Changsha, Hunan 410011; ${ }^{3}$ Cancer Research Institute, \\ Xiangya School of Medicine, Central South University, Changsha, Hunan 410078, P.R. China
}

Received April 25, 2016; Accepted July 27, 2017

DOI: $10.3892 /$ etm.2017.5495

\begin{abstract}
The Epstein-Barr virus (EBV) lytic cycle contributes to the development of EBV-associated diseases. EBV-encoded latent membrane protein 1 (LMP1) is key to EBV lytic replication, and our previous work indicated that epigallocatechin-3-gallate (EGCG) inhibited constitutive EBV lytic infection through the suppression of LMP1-activated phosphoinositide 3-kinase/Akt and mitogen-activated protein kinase kinase/extracellular signal-related protein kinase 1/2 signaling. The present study demonstrated that LMP1 in CNE-LMP1 constructed cells significantly induced the expression of the EBV lytic proteins BZLF1 $(\mathrm{P}<0.001)$ and BMRF1 $(\mathrm{P}<0.05)$ compared with CNE1 cells. Following treatment with a specific DNAzyme that targets LMP1, significantly reduced protein expression levels of BZLF1 and BMRF1 in EBV-associated epithelial carcinoma CNE1-LMP1 cells $(\mathrm{P}<0.001$ and $\mathrm{P}<0.01$, respectively) and lymphoma $\mathrm{B} 95.8$ cells (both $\mathrm{P}<0.01$ ) were observed. Furthermore, EGCG significantly inhibited the mRNA and protein expression levels of LMP1 $(\mathrm{P}<0.05)$ in an apparent dose-dependent manner in CNE1-LMP1 and B95.8 cells. Thus, the present findings indicated that the molecular mechanism underlying EGCG inhibition of EBV lytic infection involves downregulation of LMP1.
\end{abstract}

\section{Introduction}

Epstein-Barr virus (EBV) is a human herpes virus that infects over $90 \%$ of the human population worldwide (1). EBV is

Correspondence to: Dr Ya Cao, Key Laboratory of Carcinogenesis and Cancer Invasion, Chinese Ministry of Education, Xiangya Hospital, Central South University, 110 Xiangya Road, Changsha, Hunan 410078, P.R. China

E-mail: ycao98@vip.sina.com

Key words: epigallocatechin-3-gallate, Epstein-Bar virus, latent membrane protein 1 , lytic infection suggested to be an environmental factor associated with the development of several human malignancies, including nasopharyngeal carcinoma (NPC), Burkitt's lymphoma, Hodgkin's disease (HD), gastric cancer, natural killer/T-cell lymphoma, acquired immune deficiency syndrome- and transplantation-associated lymphoma $(2,3)$, breast carcinoma, and hepatocellular carcinoma (4,5). EBV, as all other herpes viruses, may establish a latent or lytic infection in host cells (6). Notably, studies suggest that EBV reactivation into a lytic cycle may contribute to the pathogenesis of malignancies $(7,8)$. EBV lytic infection in vivo has been identified by elevated antibody titers against EBV lytic antigens and by increased viral DNA load in the serum/plasma, and these observations correspond with advanced cancer stages, poor prognosis or tumor recurrence following therapy $(9,10)$. Additionally, serological studies have indicated that EBV lytic infection may occur months or years prior to a clinical diagnosis of NPC, HD or Burkitt's lymphoma, which suggests EBV lytic infection may be a risk factor for cancer development (11-13).

Green tea contains (-)-epigallocatechin-3-gallate (EGCG), which is reported to have antioxidant, antibacterial and antitumor effects $(14,15)$. EGCG has been indicated to modulate multiple signaling pathways, including the phosphoinositide 3-kinase (PI3-K)/Akt and mitogen-activated protein kinase (MAPK) signaling pathways, which may enable it to exert its cancer chemopreventive and therapeutic effects $(16,17)$.

EBV encoded latent membrane protein 1 (LMP1), which is considered to have oncogenic properties, has been identified in $90 \%$ of patients with NPC $(18,19)$. Through its cytoplasmic C-terminal, LMP1 may trigger multiple signal transduction cascades, including the MAPK kinase (MEK)/extracellular signal-regulated kinase (ERK), PI3-K/Akt, c-Jun N-terminal kinase (JNK) and signal transducer and activator of transcription 3 signaling pathways, to alter cell growth and survival $(20,21)$. Furthermore, LMP1 is established as a critical viral protein required for the EBV life cycle (13,22-25).

Our previous work indicated that EGCG may inhibit the spontaneous reactivation of $\mathrm{EBV}$, which was associated with activation of the MAPK and PI3-K/Akt pathways (26). 
Furthermore, EGCG has been reported to modulate signal pathways induced by LMP1 (16). Therefore, the present study investigated whether EGCG can suppress EBV lytic infection by inhibiting LMP1 expression.

\section{Materials and methods}

Cell lines and culture. B95.8, an EBV-positive marmoset B cell line, was obtained from the American Type Culture Collection (Manassas, VA, USA) and preserved at the Cancer Research Institute, Xiangya School of Medicine (Central South University, Changsha, China) (27). CNE1 is an LMP1-negative EBV-associated epithelial carcinoma cell line that has been identified to be cross-contaminated with HeLa cells and an additional cell line of unknown origin (28). The CNE1-LMP1 cell line, which stably expresses LMP1, was obtained from the Cancer Research Institute of Central South University $(26,29)$. All these cells were also preserved at the Cancer Research Insitute at Xiangya School of Medicine (26,27,29,30). All cells were cultured in Roswell Park Memorial Institute (RPMI)-1640 medium (Gibco; Thermo Fisher Scientific, Inc., Waltham, MA, USA) containing $10 \%$ fetal bovine serum (FBS; Invitrogen; Thermo Fisher Scientific, Inc.) and $100 \mathrm{U} / \mathrm{ml}$ penicillin/streptomycin, and were maintained at $37^{\circ} \mathrm{C}$ and $99 \%$ humidity, in an atmosphere containing $5 \% \mathrm{CO}_{2}$.

Plasmids and transient transfection. An expression vector for wild-type LMP1, pSG5-LMP1, was derived from the B95.8 EBV strain and provided by Dr Lzumi (Brigham and Women's Hospital, Boston, MA, USA) (30). CNE1 cells $\left(5 \times 10^{5}\right.$ cells/well) were transfected with different concentrations of pSG5-LMP1 plasmid (0, 0.5 and $1 \mu \mathrm{g} /$ well) or with control pSG5 vector (Agilent Technologies, Inc., Santa Clara, CA, USA; $1 \mu \mathrm{g} /$ well) using Lipofectamine 2000 (Invitrogen; Thermo Fisher Scientific, Inc.). Lipofectamine-DNA complexes were incubated with cells at $37^{\circ} \mathrm{C}$ for $4 \mathrm{~h}$ in RPMI 1640 , then washed in PBS and maintained in RPMI supplemented with $10 \%$ FBS and $100 \mathrm{U} / \mathrm{ml}$ penicillin/streptomycin at $37^{\circ} \mathrm{C}$ under $5 \% \mathrm{CO}_{2}$ and $99 \%$ humidity for $24 \mathrm{~h}$, prior to being harvested for western blot analysis.

DNAzyme (DZ1) and transfection. B95.8, CNE1-LMP1 and CNE1 cells $\left(5 \times 10^{5}\right.$ cells/well) were seeded in 6 -well plates at $37^{\circ} \mathrm{C}$ in an atmosphere containing $5 \% \mathrm{CO}_{2}$ overnight. $\mathrm{DZI} /$ tetra (4-methylpyridyl) porphyrine mixtures were made at a charge ratio of 1 and with $2 \mu \mathrm{M}$ DZ1 oligonucleotides or control oligonucleotides (ODN). The EBV LMP1-targeted DNAzyme DZ1 is an LMP1-targeted DNAzyme that binds and cleaves LMP1 RNA in a highly sequence-specific manner and was synthesized by Oligos Etc., Inc. (Wilsonville, OR, USA). Tetra (4-methylpyridyl) porphyrine was purchased from Frontier Scientific, Inc. (Logan, UT, USA) (31). Control oligonucleotides (Takara Biotechnology Co., Ltd., Dalian, China) were designed by inverting the catalytic core sequence, as described previously (31-33). Mixtures were incubated for $15 \mathrm{~min}$ at room temperature to form transfection complexes. Cells were rinsed twice with phosphate buffered saline and then incubated with the transfection mixtures of either DZ1 or ODN at $37^{\circ} \mathrm{C}$ for $4 \mathrm{~h}$ in an atmosphere containing $5 \% \mathrm{CO}_{2}$, which was followed by the addition of complete medium to the wells. Cells were subsequently incubated at $37^{\circ} \mathrm{C}$ for $24 \mathrm{~h}$ in an atmosphere containing $5 \% \mathrm{CO}_{2}$.

EGCG and cell treatment. EGCG was purchased from Sigma-Aldrich (Merck KGaA; Darmstadt, Germany) and prepared in autoclaved water as a stock solution for in vitro experiments. Prior to treatment with different concentrations of $\operatorname{EGCG}(0,5,10$ or $20 \mu \mathrm{M})$ at $37^{\circ} \mathrm{C}$ for $24 \mathrm{~h}, \mathrm{CNE} 1-\mathrm{LMP} 1$ and B95.8 cells ( $2 \times 10^{5}$ cells/well) were starved in RPMI-1640 supplemented with $0.1 \% \mathrm{FBS}$ at $37^{\circ} \mathrm{C}$ in an atmosphere containing $5 \% \mathrm{CO}_{2}$ for $24 \mathrm{~h} . \mathrm{H}_{2} \mathrm{O}$ treatment was used as a negative control ( $0 \mu \mathrm{M}$ EGCG treatment group).

Preparation of cell lysates and western blot analysis. Whole cell lysate preparation and western blot analysis were performed according to published methods (26). EGCG-treated B95.8 and CNE1-LMP1 cells, or post-transfection B95.8, CNE1-LMP1 and CNE1 cells were harvested at the indicated time, lysed in lysis buffer [10 mM Tris-HCl, pH 8.0, 1 mM EDTA, 2\% SDS, $5 \mathrm{mM}$ dithiothreitol, $10 \mathrm{mM}$ phenylmethyl sulfonylfluoride, $1 \mathrm{mM} \mathrm{Na}_{3} \mathrm{VO}_{4}, 1 \mathrm{mM} \mathrm{NaF}$ and $10 \%$ (v/v) glycerol; and a protease inhibitor cocktail tablet (Roche Diagnostics, Basel, Switzerland)], incubated on ice for 30 min with mixing every $10 \mathrm{~min}$, and subsequently centrifuged for $10 \mathrm{~min}$ at $16,800 \mathrm{xg}$ and $4^{\circ} \mathrm{C}$. Supernatant was collected as whole cell lysates and the protein concentration was measured using a BCA Assay Reagent (Pierce; Thermo Fisher Scientific, Inc.). Protein samples $(50 \mu \mathrm{g} / \mathrm{lane})$ were separated by $6-12 \%$ SDS-PAGE, transferred onto a nylon membrane. The membranes were blocked with buffer containing 5\% non-fat milk in PBS with $0.05 \%$ Tween-20 (PBST) at room temperature for $2 \mathrm{~h}$, and incubated with different primary antibodies overnight at $4^{\circ} \mathrm{C}$. The following primary antibodies were used for immuno-detection: Mouse BZLF1 monoclonal antibody (SC-53904; Santa Cruz Biotechnology, Inc., Dallas, TX, USA) at 1:200 dilution; EBV nuclear antigen 1 (EBNA1) antibody (ab25653; Abcam; Cambridge, UK) at 1:1,000 dilution; BMRF1 antibody (ab6524; Abcam), which binds to BMRF1 (Ea-D) p52/50 of EBV, at 1:1,000 dilution; $\beta$-actin (Ac-15; Sigma-Aldrich; Merck KGaA) at 1:2,000 dilution; and LMP1 monoclonal antibody (M0897; Dako; Agilent Technologies, Inc.), which binds to full length LM1 $(62 \mathrm{kDa})$ and truncated LMP1 (42 kDa) at 1:200 dilution. Following a second wash with PBST, the membranes were incubated with anti-mouse (sc-2005; Santa Cruz Biotechnology, Inc.) horseradish peroxidase-conjugated secondary antibody for $1 \mathrm{~h}$ at room temperature, and color was subsequently developed using an enhanced chemiluminescence detection kit (Pierce; Thermo Fisher Scientific, Inc.). The protein bands were visualized following exposure of the membranes to Kodak X-ray film. Densitometric analysis of the bands was carried out using ImageJ software 1.42q (National Institutes of Health, Bethesda, MD, USA).

Reverse transcription-quantitative polymerase chain reaction (RT-qPCR). Total RNA from EGCG-treated B95.8 and CNE1-LMP1 cells was isolated using TRIzol reagent (Invitrogen; Thermo Fisher Scientific, Inc.) and complementary DNA was synthesized according to a previously published method (26). LMP1 expression was measured by RT-qPCR with the following primers: $L M P 1$, forward, 5'-ATA 
CCTAAGACAAGTAAGCA-3' and reverse 5'-ACACACTGC CCTGAGGATGG-3' (34); The PCR products underwent electrophoresis on $2.5 \%$ agarose gel. Visualization following ethidium bromide staining at room temperature for $30 \mathrm{~min}$ was performed under UV light. qPCR was performed using a Rotor-Gene 6000 thermocycler (Qiagen GmbH, Hilden, Germany) and SYBR Premix Ex Taq II (Takara Biotechnology Co., Ltd.) and $2 \mu \mathrm{l}$ complementary DNA with the following primers: $L M P 1$, forward 5'-TGACTGGACTGGAGGAGC-3' and reverse 5'-AGCGATGAGCAGGAGGGT - 3 '; and $\beta$-actin, forward 5'-TTCCAGCCTTCCTTCCTGGG-3' and reverse 5'-TTGC GCTCAGGAGGAGCAAT-3'. The following thermocycling conditions were used: Initial denaturation at $95^{\circ} \mathrm{C}$ for $1 \mathrm{~min}$, followed by 40 cycles of denaturing at $95^{\circ} \mathrm{C}$ for $15 \mathrm{sec}$, annealing at $55^{\circ} \mathrm{C}$ for $20 \mathrm{sec}$ and extension at $72^{\circ} \mathrm{C}$ for $30 \mathrm{sec}$, and a final extension at $72^{\circ} \mathrm{C}$ for $10 \mathrm{~min}$ with subsequent cooling to $4^{\circ} \mathrm{C}$. Relative mRNA abundance was calculated by the $2^{-\Delta \Delta C q}$ method using $\beta$-actin as the internal control (35). For each experiment, the mRNA levels in untreated cells were used as controls and set as 1 . The mRNA expression levels were represented relative to those in the untreated cells.

Statistical analysis. Data were analyzed using GraphPad Prism 5 (GraphPad Software, Inc., La Jolla, CA, USA). All values were expressed as the mean \pm standard error of the mean of triplicate experiments. Two-group comparisons were performed using Student's t-tests, and $\mathrm{P}<0.05$ was considered to indicate a statistically significant difference.

\section{Results}

$L M P 1$ enhances the expression levels of EBV lytic proteins in CNE1 cells. Our previous work indicated that EBV lytic and latent infection proteins are present in NPC tumor cells and lymphomas (26). In the present study, EBV latent and lytic protein expression levels were investigated in the B95.8 and CNE1 cell lines and compared with the constructed CNE1-LMP1 cells. B95.8 cells were considered as a positive control as the line is permissive for viral lytic replication (36). Western blot analysis revealed that the EBV latent proteins, EBNA1 and LMP1 (37), were constitutively expressed in B985.8, CNE1 and CNE1-LMP1 cell lines, which indicated that these cell lines were EBV-positive (Fig. 1A). Additionally, the constructed CNE1-LMP1 cell line that constitutively expressed LMP1 exhibited markedly increased expression levels of the EBV lytic proteins BZLF1 and BMRF1 $(37,38)$ when compared with the CNE1 control cells (Fig. 1B). As depicted in Fig. 1C, the differences in the protein levels of BZLF1 and BMRF1 between the CNE1-LMP1 and CNE1 cells were determined to be statistically significant $(\mathrm{P}<0.001$ and $\mathrm{P}<0.05$, respectively).

To further evaluate the expression of the EBV lytic proteins, CNE1 cells were transiently transfected with the LMP1-expressing pSG5-LMP1 plasmid. As indicated in Fig. 1D and E, pSG5-LMP1 in the CNE1 cells increased the protein levels of BZLF1 and BMRF1 protein in an apparent dose-dependent manner. Furthermore, compared with the CNE1 cells treated with pSG5 or $0 \mu \mathrm{g}$ pSG5-LMP1, the CNE1 cells treated with $1 \mu \mathrm{g}$ pSG5-LMP1 exhibited significantly increased protein levels of BZLF1 and BMFR1 ( $<<0.05$; Fig. 1E).
Inhibition of LMPI expression decreases the expression of EBV lytic proteins in LMPI-positive cells. To clarify the potential regulatory effect of LMP1 on EBV lytic protein expression, DZ1 was used to downregulate LMP1 in B95.8 and CNE1-LMP1 cells (Fig. 2). As expected, LMP1 protein expression was significantly downregulated in the CNE1-LMP1 and B95.8 cells following DZ1 treatment when compared with the respective ODN controls $(\mathrm{P}<0.001$ and $\mathrm{P}<0.01$, respectively). More notably, following DZ1 treatment, the CNE1-LMP1 and B95.8 cells exhibited significantly reduced protein levels of BZLF1 ( $\mathrm{P}<0.001$ and $\mathrm{P}<0.01$, respectively) and BMRF1 (both $\mathrm{P}<0.01$ ). ODN had no significant effect on the protein levels of LMP1 or EBV lytic proteins when compared with the untreated control cells.

Effect of LMP-1-specific DZ1 on the expression of EBV lytic proteins in LMPI-negative cells. To ascertain the specific cytotoxicity of DZ1, LMP1-negative CNE1 cells were treated with $2 \mu \mathrm{M}$ LMP1-targeting DZ1. As depicted in Fig. 3, in LMP1-negative CNE1 cells, DZ1 had no significant effect on the expression of the EBV lytic proteins BZLF1 and BMRF1. Thus, DZ1 was indicated to specifically inhibit LMP1 expression and consequently EBV lytic protein expression in the CNE1-LMP1 cells. These findings also verified that LMP1 had a positive regulatory effect on the expression of the EBV lytic proteins BZLF1 and BMRF1.

Inhibitory effect of EGCG on the expression of LMPI. Our previous work indicated that EGCG exerted inhibitory effects on the viability of CNE1-LMP1 and B95.8 cells $\left(\mathrm{IC}_{50}\right.$ $20 \mu \mathrm{M}$ ), and that this was dose- and time-dependent (26). Thus, EGCG $(0-20 \mu \mathrm{M})$ was used in the current experiments. To determine whether EGCG affected the protein expression of LMP1, LMP1-positive CNE1-LMP1 and B95.8 cells were treated with EGCG $(0,5,10$ and $20 \mu \mathrm{M})$, after which the cells were harvested and the levels of LMP1 expression were measured by western blot analysis. As depicted in Fig. 4A, the protein levels of LMP1 in CNE1-LMP1 and B95.8 cells treated with EGCG were markedly reduced, mostly notably following treatment with $20 \mu \mathrm{M}$ EGCG, when compared with those in the untreated CNE1-LMP1 and B95.8 cells. Subsequent quantification of the results indicated that these differences in the levels of LMP1 were statistically significant; in the CNE1-LMP1 cells, the protein levels of LMP1 were deemed to be significantly decreased following treatment with $10(\mathrm{P}<0.05)$ and $20 \mu \mathrm{M}(\mathrm{P}<0.01)$ EGCG, while for the B95.8 cells, the protein levels of LMP1 were significantly decreased following treatment with $5(\mathrm{P}<0.01), 10(\mathrm{P}<0.01)$ and $20 \mu \mathrm{M}(\mathrm{P}<0.001)$ EGCG (Fig. 4B).

Decreased protein expression may be due to downregulated mRNA; therefore, the mRNA levels of LMP1 in the CNE1-LMP1 and B95.8 cells were determined. The results indicated that EGCG treatment markedly downregulated LMP1 mRNA in the CNE1-LMP1 and B95.8 cells (Fig. 4C). Quantification of these results indicated that EGCG treatment $(5-20 \mu \mathrm{M})$ significantly downregulated LMP1 mRNA in the CNE1-LMP1 and B95.8 cells $(\mathrm{P}<0.05)$ in an apparent dose-dependent manner, (Fig. 4D). These findings suggested that EGCG decreased viral LMP1 expression in the EBV-associated epithelial carcinoma cell line CNE1-LMP1 
A

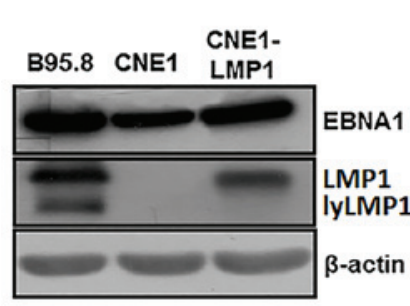

$\mathbf{B}$

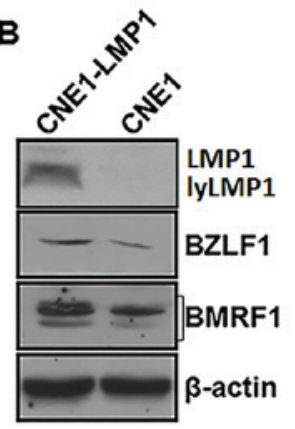

C

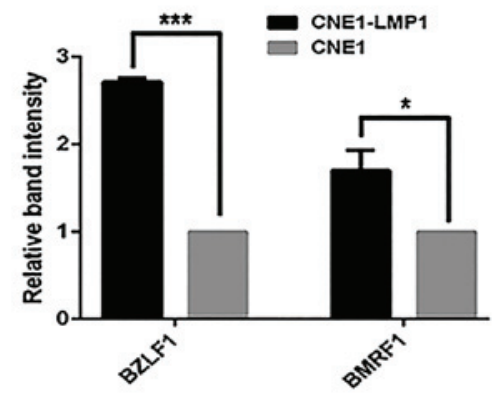

D

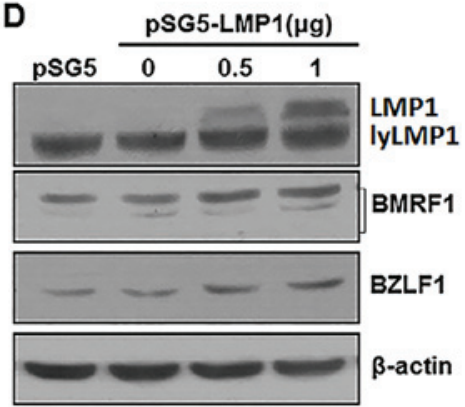

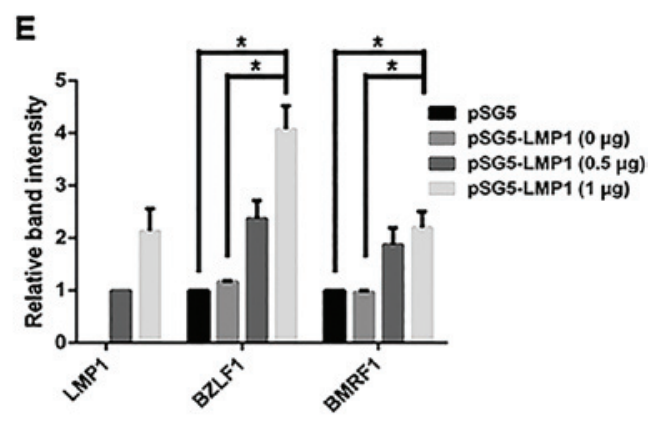

Figure 1. LMP1 enhanced the protein expression of the EBV lytic proteins BZLF1 and BMRF1. (A) B95.8, CNE1 and constructed CNE1-LMP1 cells were lysed and western blot analysis was performed to measure the protein levels of EBNA1 and LMP1 in each cell type. $\beta$-actin was used as the loading control (B) The protein levels of LMP1 and the EBV lytic proteins BZLF1 and BMRF1 in CNE1-LMP1 and CNE1 cells were determined by western blot analysis and compared with $\beta$-actin levels. (C) The protein levels of the EBV lytic proteins were analyzed by densitometry and presented as a ratio relative to the loading control $\beta$-actin. (D) CNE1 cells were transiently transfected with different quantities of LMP1-expressing plasmid ( $0,0.5$ and $1 \mu \mathrm{g})$ for $24 \mathrm{~h}$ and western blot analysis was performed to measure the protein levels of BZLF1, BMRF1, LMP1 and $\beta$-actin. (E) The levels of LMP1, BZLF1 and BMRF1 protein estimated by densitometry were presented as a ratio relative to the loading control $\beta$-actin. Values were presented as the mean \pm standard deviation of triplicate experiments. ${ }^{*} \mathrm{P}<0.05$ and ${ }^{* * * *} \mathrm{P}<0.001$. LMP1, latent membrane protein 1; lyLMP1, lytic latent membrane protein 1; EBV, Epstein-Barr virus.
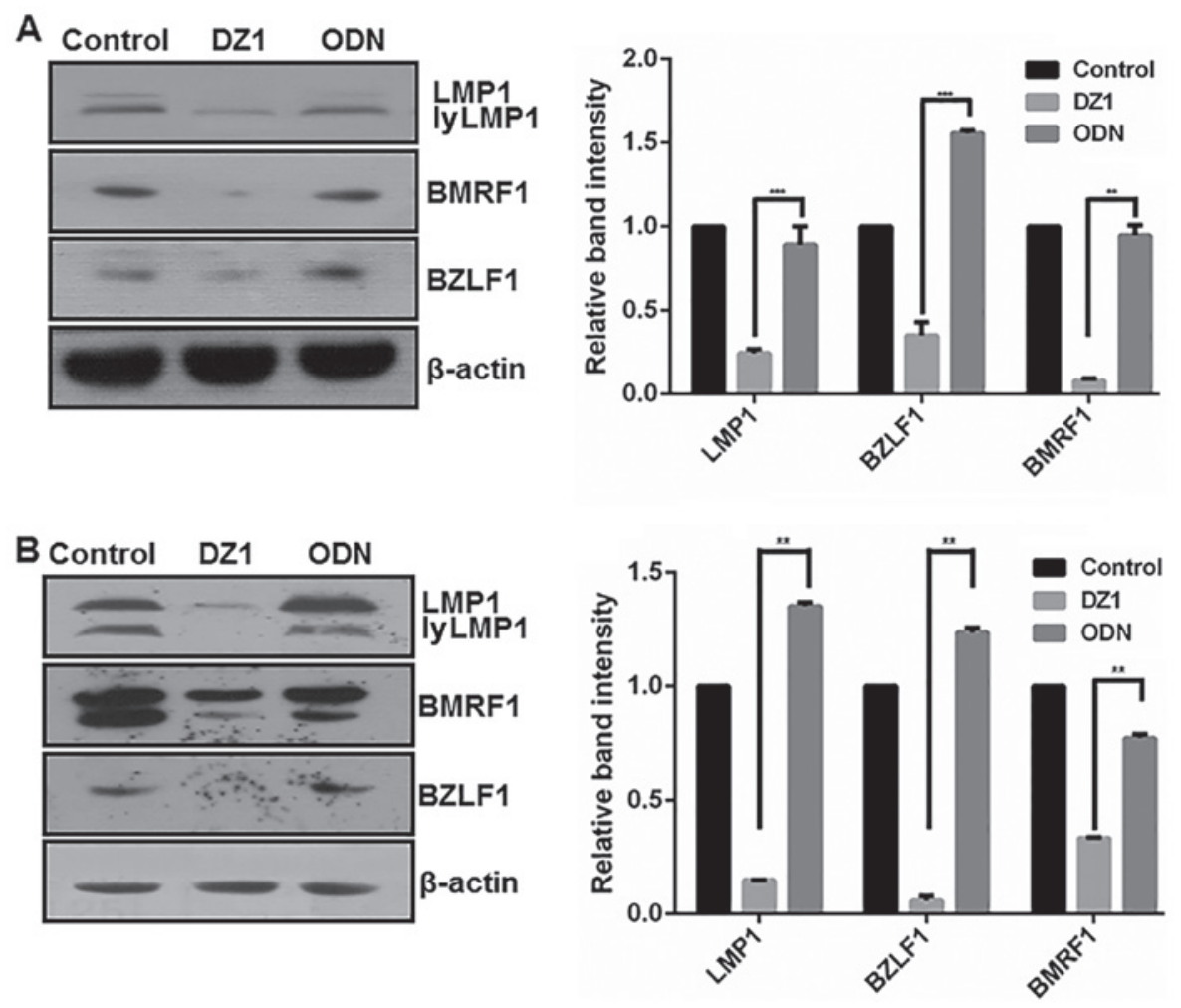

Figure 2. Reduced LMP1 expression induced by LMP1-specific DZ1 treatment was associated with downregulation of the EBV lytic proteins BZLF1 and BMRF1 in LMP1-positive cells. (A) CNE1-LMP1 and (B) B95.8 cells were transfected with DZ1 or control ODN, and subjected to western blot analysis. The protein levels of LMP1, BZLF1 and BMRF1 in CNE1-LMP1 and B95.8 cells were measured by densitometry and presented as a ratio relative to the loading control $\beta$-actin. Untreated cells were considered as controls and set as 1. Cells treated with ODN were used as transfection mimics. Values were presented as the mean \pm standard deviation of triplicate experiments. ${ }^{* *} \mathrm{P}<0.01$ and ${ }^{* * *} \mathrm{P}<0.001$. LMP1, latent membrane protein 1; lyLMP1, lytic latent membrane protein 1 ; EBV, Epstein-Barr virus; DZ1, DNAzyme; ODN, oligonucleotide. 

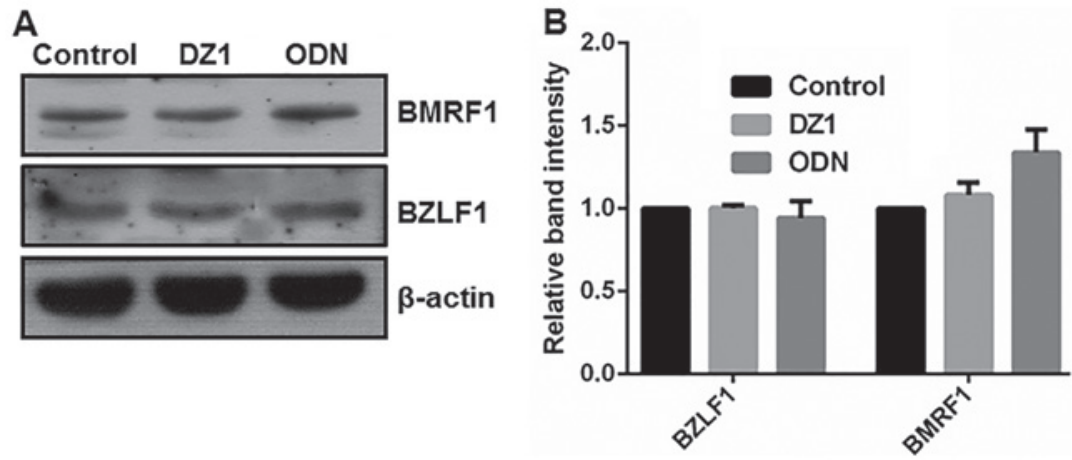

Figure 3. Effect of LMP1-specific DZ1 treatment on EBV lytic protein expression in LMP1-negative cells. (A) The protein levels of BZLF1 and BMRF1 were detected in CNE1 cells. (B) The protein levels were measured using densitometry and presented as a ratio relative to $\beta$-actin. Untreated cells were used as controls and set at 1 . Cells treated with ODN were used as transfection mimics. Values were presented as the mean \pm standard deviation of triplicate experiments. LMP1, latent membrane protein 1; EBV, Epstein-Barr virus; DZ1, DNAzyme; ODN, control oligonucleotide.
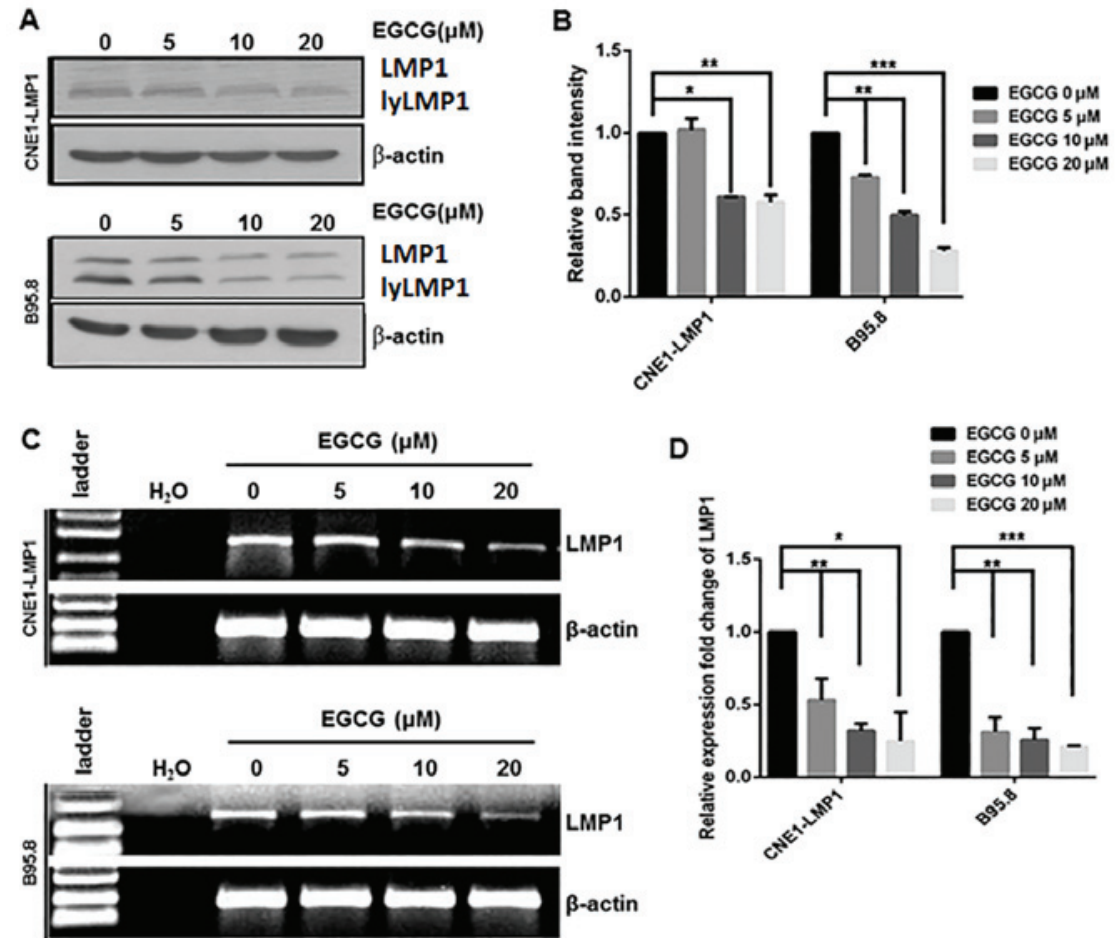

Figure 4. EGCG inhibited the protein expression of LMP1 in CNE1-LMP1 and B95.8 cells. (A) Western blot analysis was performed to detect the protein levels of LMP1 in CNE1-LMP1 and B95.8 cells following treatment with 0, 5, 10 and $20 \mu$ M EGCG. (B) Protein expression was measured by densitometry and presented as a ratio relative to the loading control $\beta$-actin. Untreated cells were used as controls and set at 1 . (C) The effect of EGCG treatment $(0,5,10$ and $20 \mu \mathrm{M})$ on the mRNA expression of LMP1 was determined using reverse transcription-quantitative polymerase chain reaction. $\mathrm{H}_{2} \mathrm{O}$ treatment was used as a negative control ( $0 \mu \mathrm{M}$ EGCG treatment group) and $\beta$-actin was used as a loading control. (D) LMP1 mRNA expression was quantified; untreated cells were used as controls and set at 1 . Values were presented as the mean \pm standard deviation of triplicate experiments. ${ }^{*} \mathrm{P}<0.05,{ }^{* *} \mathrm{P}<0.01$ and ${ }^{* * *} \mathrm{P}<0.001$. EGCG; epigallocatechin-3-gallate; LMP1, latent membrane protein 1; lyLMP1, lytic latent membrane protein 1.

and EBV-positive B95.8 cell line at both the protein and mRNA level.

\section{Discussion}

The life cycle of EBV includes latent and lytic stages. In the majority of asymptomatic carriers, the lytic cycle of EBV in the host cells is periodically reactivated (7). Previous studies have focused on the effects of EBV latent infection $(2,20)$. Other studies have demonstrated that the lytic cycle of EBV was able to increase B-cell transformation efficiency at cell culture levels and the development of B-cell lymphoma in a humanized mouse model (39-41). Our previous data demonstrated that EBV lytic infection proteins are present, not only in CNE1-LMP1 and lymphoma cell lines, but also in patient biopsies (26). Similarly, the present study identified markers of the EBV lytic cycle in EBV-associated epithelial carcinoma and lymphoma cell lines.

LMP1 is an EBV-encoded 62-kDa integral membrane oncogenic protein containing 386 amino acid residues that is primarily composed of a short intracellular N-terminus, six hydrophobic transmembrane domains and an intracellular 
C-terminus that includes the three functional domains CTAR1, CTAR2 and CTAR3 (42). LMP1 has been be detected in $90 \%$ of patients with NPC $(18,19)$. Through its cytoplasmic C-terminus, LMP1 has been reported to trigger multiple signal transduction cascades associated with the EBV lytic cycle, including MEK/ERK, PI3-K/Akt, nuclear factor- $\kappa \mathrm{B}$ and JNK signaling pathways $(20,21)$. In addition, the LMP1 gene of several EBV strains also contains a late lytic cycle promoter, EDL1A, which drives the expression of an amino-terminally truncated form of LMP1, lytic LMP1 (lyLMP1). lyLMP1, has 258 amino acid residues $\sim 42-\mathrm{kD}$, expressed in the lytic phase (43). Modulation of LMP1-activated signaling pathways was the first identified biological activity associated with lyLMP1, and this activity may contribute to the progression of EBV's lytic cycle $(43,44)$. The present findings also suggested that LMP1 and lyLM1 were expressed in B95-8 and CNE1-LMP1 cells and that there was spontaneous reactivation of EBV in the cell lines.

A number of studies have demonstrated that LMP1 serves an important role in the EBV lytic cycle $(24,45,46)$. While LMP1 may be expressed in some states of EBV latency, significant induction of full-length LMP1 is frequently observed during virus reactivation into the lytic cycle $(13,47,48)$. Notably, when EBV reactivation is induced by various stimuli, including cross-linking of surface immunoglobulin, virus superinfection and treatment with phorbol ester, 5-azacytidine, butyrate or histone deacetylase inhibitors, the expression of full-length LMP1 may be significantly increased (13,22-25). Furthermore, transfection of cells with an exogenous Rta plasmid has been demonstrated to induce the expression of LMP1 in a variety of epithelial cell lines such as NPC NA, EBV-infected HeLa, 293 , P3HR1 and Akata cells (24). The close correlation between the inducible increased expression of LMP1 and the EBV replication cycle indicates that LMP1 expression, as a lytic cycle gene, is under the control of the lytic cycle program (24). In a previous study, lack of LMP1 expression severely impaired virus release into culture supernatants, which resulted in poor infection efficiency. These results have suggested that LMP1 serves an important role in EBV particle release from cells during the lytic cycle and in the infection of new host cells (46). Furthermore, a different study identified that low expression of LMP1 suppressed the activity of the EBV latent replication origin oriP, and that the LMP1 binding site for tumor necrosis factor receptor-associated factor was essential for this suppressive effect (49).

In the present study, the protein expression levels of the EBV lytic proteins BZLF1 and BMRF1 in the LMP1-positive CNE1-LMP1 cells were significantly elevated when compared with the LMP1-negative CNE1 cells. Through an induction strategy with an LMP1 expression plasmid to induce LMP1 expression and a blockade strategy with DZ1 to inhibit LMP1 expression, it was indicated that LMP1 promoted the expression of the EBV lytic proteins BZLF1 and BMRF1. To ascertain the specific cytotoxicity of DZ1, LMP1-negative CNE1 cells were used as an experimental model, in which it was demonstrated that DZ1 had no significant effect on the expression of BZLF1 and BMRF1. These findings suggest a positive regulatory effect of LMP1 on the expression of the EBV lytic proteins BZLF1 and BMRF1.

EBV lytic genes, including BZLF1 and BMRF1, or cellular genes induced by these viral lytic proteins, may encode paracrine factors that promote tumor growth (39). It has been reported that BZLF1 has various malignancy-promoting activities (50). Therefore, EBV lytic infection may be a notable factor to consider in malignant transformation, as EBV infection results in changes to the infected host cells or nearby cells (45), and the biological characteristics of these cells may be altered in a way that may increase the degree of malignancy and promote the occurrence of metastasis (51). Furthermore, LMP1 is an important viral protein required for the EBV lytic life cycle (45). Therefore, selection of this protein for augmenting virus release may be a critical evolutionary step for EBV.

Other studies have suggested a conflicting model in which LMP1 inhibits lytic cycle progression (52-54). For instance, LMP1 was reported to inhibit lytic cycle induction via the transcription factor nuclear factor $-\kappa \mathrm{B}$ in an EBV-positive Burkitt's lymphoma P3HR1-c16 cell line, which lacks LMP1 and may be activated into a virally productive lytic cycle (52). These findings indicate that in B cells, EBV self-limits its lytic cycle via the transcription factor nuclear factor $(\mathrm{NF})-\kappa \mathrm{B}$. In addition, LMP1 inhibits lytic cycle progress via two distinct $\mathrm{NF}-\kappa \mathrm{B}$-independent mechanisms: One associated with the cytosolic C-terminal activating regions and the other with the transmembrane region of LMP1 (52). Additionally, cluster of differentiation (CD) 40-CD40 ligand interactions and viral mimics of activated CD40 and LMP1 suppress virus reactivation, and this regulation of latency by CD40 and LMP1 may have important implications for the balance between EBV and its host in normal or immunocompromised individuals (55). The discrepancy between these previous findings with the present results may be due to differences in the experimental systems and thus expression levels of LMP1 protein. Regardless, the above findings indicate that LMP1 may serve dual roles in EBV lytic replication.

LMP1 may create an optimum cellular environment for efficient EBV DNA replication by promoting cell proliferation or triggering necessary signaling pathways $(2,20)$. The latent form of infection allows the virus to persist for the lifetime of the host, whereas the lytic form of infection enables infectious virion production and transmission from cell to cell and from host to host. Both forms of infection are essential for the long-term success of the virus (45). It is also speculated that once EBV is reactivated into the lytic cycle, the induced expression of LMP1 is considered to be critical for efficient virus release and infection of new host cells. However, high levels of LMP1 may negatively regulate EBV lytic infection (52-54). Therefore, EBV has developed a series of strategies to maintain itself in host cells over the host's lifetime, and only periodically produces infectious virons to transmit and infect new host cells.

Previous studies on cancer chemoprevention using EGCG have suggested that EGCG has anti-carcinogenic activity in various organs in animal models $(14,56)$. Alternative studies and our previous and present studies indicate that EGCG inhibits EBV lytic infection, though the mechanism is not well understood (26,57-59). In our previous study, CNE1-LMP1 and B95.8 cells with EBV spontaneous lytic replication were used to mimic the natural state of infected cells, and it was indicated that EGCG inhibited EBV spontaneous lytic replication by inhibiting activation of MEK/ERK1/2 and 
PI3-K/Akt signaling (26). Considering that EGCG may modulate signaling pathways induced by LMP1 (16), the present study investigated the biological significance of EGCG on LMP1 expression during the lytic cycle of viral replication, and observed that EGCG inhibited the expression of LMP1 at the transcriptional and translational levels. Thus, EGCG may inhibit EBV spontaneous lytic replication by a novel mechanism involving the inhibition of LMP1 expression. Further elucidation of the molecular mechanisms underlying EGCG activity during EBV lytic replication may facilitate the development of therapies for EBV-positive malignancies.

\section{Acknowledgements}

The present study was supported by the National Nature Science Foundation of China (grant no. 81101474).

\section{References}

1. Cohen JI, Fauci AS, Varmus H and Nabel GJ: Epstein-Barr virus: An important vaccine target for cancer prevention. Sci Transl Med 3: 107fs7, 2011.

2. Young LS and Murray PG: Epstein-Barr virus and oncogenesis: From latent genes to tumours. Oncogene 22: 5108-5121, 2003.

3. Young LS and Rickinson AB: Epstein-Barr virus: 40 years on. Nat Rev Cancer 4: 757-768, 2004.

4. Sugawara Y, Makuuchi M and Takada K: Detection of Epstein-Barr virus DNA in hepatocellular carcinoma tissues from hepatitis C-positive patients. Scand J Gastroenterol 35: 981-984, 2000

5. Kutok JL and Wang F: Spectrum of Epstein-Barr virus-associated diseases. Annu Rev Pathol 1: 375-404, 2006.

6. Hammerschmidt W and Sugden B: Replication of Epstein-Barr viral DNA. Cold Spring Harb Perspect Biol 5: a013029, 2013.

7. Murata T: Regulation of Epstein-Barr virus reactivation from latency. Microbiol Immunol 58: 307-317, 2014.

8. Murata T and Tsurumi T: Switching of EBV cycles between latent and lytic states. Rev Med Virol 24: 142-153, 2014

9. Lei KI, Chan LY, Chan WY, Johnson PJ and Lo YM: Quantitative analysis of circulating cell-free Epstein-Barr virus (EBV) DNA levels in patients with EBV-associated lymphoid malignancies. Br J Haematol 111: 239-246, 2000.

10. Lo YM: Quantitative analysis of Epstein-Barr virus DNA in plasma and serum: Applications to tumor detection and monitoring. Ann N Y Acad Sci 945: 68-72, 2001.

11. Geser A, de Thé G, Lenoir G, Day NE and Williams EH: Final case reporting from the Ugandan prospective study of the relationship between EBV and Burkitt's lymphoma. Int J Cancer 29: 397-400, 1982

12. Mueller N, Evans A, Harris NL, Comstock GW, Jellum E, Magnus K, Orentreich N, Polk BF and Vogelman J: Hodgkin's disease and Epstein-Barr virus. Altered antibody pattern before diagnosis. N Engl J Med 320: 689-695, 1989.

13. BoosH,BergerR,Kuklik-Roos C,IftnerTandMueller-LantzschN Enhancement of Epstein-Barr virus membrane protein (LMP) expression by serum, TPA, or n-butyrate in latently infected Raji cells. Virology 159: 161-165, 1987.

14. Ramos S: Cancer chemoprevention and chemotherapy: Dietary polyphenols and signalling pathways. Mol Nutr Food Res 52: 507-526, 2008

15. Yang CS and Wang H: Cancer therapy combination: Green tea and a phosphodiesterase 5 inhibitor? J Clin Invest 123: 556-558, 2013.

16. Zhao Y, Wang $\mathrm{H}$, Zhao XR, Luo FJ, Tang $\mathrm{M}$ and Cao $\mathrm{Y}$ : Epigallocatechin-3-gallate interferes with EBV-encoding AP-1 signal transduction pathway. Zhonghua Zhong Liu Za Zhi 26 393-397, 2004 (In Chinese).

17. Kanwar J, Taskeen M, Mohammad I, Huo C, Chan TH and Dou QP: Recent advances on tea polyphenols. Front Biosci (Elite Ed) 4: 111-131, 2012

18. Tsao SW, Tramoutanis G, Dawson CW, Lo AK and Huang DP The significance of LMP1 expression in nasopharyngeal carcinoma. Semin Cancer Biol 12: 473-487, 2002
19. Dawson CW, Port RJ and Young LS: The role of the EBV-encoded latent membrane proteins LMP1 and LMP2 in the pathogenesis of nasopharyngeal carcinoma (NPC). Semin Cancer Biol 22: 144-153, 2012.

20. Zheng H, Li LL, Hu DS, Deng XY and Cao Y: Role of Epstein-Barr virus encoded latent membrane protein 1 in the carcinogenesis of nasopharyngeal carcinoma. Cell Mol Immunol 4: 185-196, 2007

21. Li H, Liu S, Hu J, Luo X, Li N, M Bode A and Cao Y: Epstein-Barr virus lytic reactivation regulation and its pathogenic role in carcinogenesis. Int J Biol Sci 12: 1309-1318, 2016.

22. Chang Y, Tung $\mathrm{CH}$, Huang YT, Lu J, Chen JY and Tsai $\mathrm{CH}$ : Requirement for cell-to-cell contact in Epstein-Barr virus infection of nasopharyngeal carcinoma cells and keratinocytes. J Virol 73: 8857-8866, 1999.

23. Contreras-Salazar B, Ehlin-Henriksson B, Klein G and Masucci MG: Up regulation of the Epstein-Barr virus (EBV)-encoded membrane protein LMP in the Burkitt's lymphoma line Daudi after exposure to n-butyrate and after EBV superinfection. J Virol 64: 5441-5447, 1990.

24. Chang Y, Lee HH, Chang SS, Hsu TY, Wang PW, Chang YS, Takada $\mathrm{K}$ and Tsai $\mathrm{CH}$ : Induction of Epstein-Barr virus latent membrane protein 1 by a lytic transactivator Rta. J Virol 78: 13028-13036, 2004.

25. Nishikawa J, Kis LL, Liu A,Zhang X, Takahara M, Bandobashi K, Kiss C, Nagy N, Okita K, Klein G and Klein E: Upregulation of LMP1 expression by histone deacetylase inhibitors in an EBV carrying NPC cell line. Virus Genes 28: 121-128, 2004.

26. Liu S, Li H, Chen L, Yang L, Li L, Tao Y, Li W, Li Z, Liu H, Tang M, et al: (-)-Epigallocatechin-3-gallate inhibition of Epstein-Barr virus spontaneous lytic infection involves ERK1/2 and PI3-K/Akt signaling in EBV-positive cells. Carcinogenesis 34: 627-637, 2013.

27. Liu SF, Wang H, Li ZJ, Deng XY, Xiang H, Tao YG, Li W, Tang M and Cao Y: Aspirin induces lytic cytotoxicity in Epstein-Barr virus-positive cells. Eur J Pharmacol 589: 8-13, 2008.

28. Strong MJ, Baddoo M, Nanbo A, Xu M, Puetter A and Lin Z: Comprehensive high-throughput RNA sequencing analysis reveals contamination of multiple nasopharyngeal carcinoma cell lines with HeLa cell genomes. J Virol 88: 10696-10704, 2014.

29. Ma X, Yang L, Xiao L, Tang M, Liu L, Li Z, Deng M, Sun L and Cao Y: Down-regulation of EBV-LMP1 radio-sensitizes nasal pharyngeal carcinoma cells via NF- $\mathrm{BB}$ regulated ATM expression. PLoS One 6: e24647, 2011.

30. Xiao L, Hu ZY, Dong X, Tan Z, Li W, Tang M, Chen L, Yang L, Tao Y, Jiang Y, et al: Targeting Epstein-Barr virus oncoprotein LMP1-mediated glycolysis sensitizes nasopharyngeal carcinoma to radiation therapy. Oncogene 33: 4568-4578, 2014.

31. Benimetskaya L, Takle GB, Vilenchik M, Lebedeva I, Miller P and Stein CA: Cationic porphyrins: Novel delivery vehicles for antisense oligodeoxynucleotides. Nucleic Acids Res 26: 5310-5317, 1998

32. Lu ZX, Ye M, Yan GR, Li Q, Tang M, Lee LM, Sun LQ and Cao Y: Effect of EBV LMP1 targeted DNAzymes on cell proliferation and apoptosis. Cancer Gene Ther 12: 647-654, 2005.

33. Cao Y, Yang L, Jiang W, Wang X, Liao W, Tan G, Liao Y, Qiu Y, Feng D, Tang F, et al: Therapeutic evaluation of Epstein-Barr virus-encoded latent membrane protein-1 targeted DNAzyme for treating of nasopharyngeal carcinomas. Mol Ther 22: 371-377, 2014.

34. Mattia E, Chichiarelli S, Hickish T, Gaeta A, Mancini C, Cunningham D and van Renswoude J: Inhibition of in vitro proliferation of Epstein Barr Virus infected B cells by an antisense oligodeoxynucleotide targeted against EBV latent membrane protein LMP1. Oncogene 15: 489-493, 1997.

35. Livak KJ and Schmittgen TD: Analysis of relative gene expression data using real-time quantitative PCR and the 2(-Delta Delta C(T)) method. Methods 25: 402-408, 2001.

36. Miller G and Lipman M: Release of infectious Epstein-Barr virus by transformed marmoset leukocytes. Proc Natl Acad Sci USA 70: 190-194, 1973

37. Tsurumi T, Fujita M and Kudoh A: Latent and lytic Epstein-Barr virus replication strategies. Rev Med Virol 15: 3-15, 2005.

38. Kenney SC and Mertz JE: Regulation of the latent-lytic switch in Epstein-Barr virus. Semin Cancer Biol 26: 60-68, 2014.

39. Katsumura KR, Maruo S and Takada K: EBV lytic infection enhances transformation of B-lymphocytes infected with EBV in the presence of T-lymphocytes. J Med Virol 84: 504-510, 2012. 
40. Kalla M, Schmeinck A, Bergbauer M, Pich D and Hammerschmidt W: AP-1 homolog BZLF1 of Epstein-Barr virus has two essential functions dependent on the epigenetic state of the viral genome. Proc Natl Acad Sci USA 107: 850-855, 2010.

41. Ma SD, Hegde S, Young KH, Sullivan R, Rajesh D, Zhou Y, Jankowska-Gan E, Burlingham WJ, Sun X, Gulley ML, et al: A new model of Epstein-Barr virus infection reveals an important role for early lytic viral protein expression in the development of lymphomas. J Virol 85: 165-177, 2011.

42. Li HP and Chang YS: Epstein-Barr virus latent membrane protein 1: Structure and functions. J Biomed Sci 10: 490-504, 2003.

43. Vazirabadi G, Geiger TR, Coffin WF III and Martin JM: Epstein-Barr virus latent membrane protein-1 (LMP-1) and lytic LMP-1 localization in plasma membrane-derived extracellular vesicles and intracellular virions. J Gen Virol 84: 1997-2008, 2003.

44. Pandya J and Walling DM: Oncogenic activity of Epstein-Barr virus latent membrane protein 1 (LMP-1) is down-regulated by lytic LMP-1. J Virol 80: 8038-8046, 2006.

45. Nawandar DM, Ohashi M, Djavadian R, Barlow E, Makielski K, Ali A, Lee D, Lambert PF, Johannsen E and Kenney SC: Differentiation-dependent LMP1 expression is required for efficient lytic Epstein-Barr virus reactivation in epithelial cells. J Virol 91: pii: e02438-16, 2017.

46. Ahsan N, Kanda T, Nagashima K and Takada K: Epstein-Barr virus transforming protein LMP1 plays a critical role in virus production. J Virol 79: 4415-4424, 2005.

47. Wang D, Liebowitz D and Kieff E: The truncated form of the Epstein-Barr virus latent-infection membrane protein expressed in virus replication does not transform rodent fibroblasts. J Virol 62: 2337-2346, 1988.

48. Rowe M, Evans HS, Young LS, Hennessy K, Kieff E and Rickinson AB: Monoclonal antibodies to the latent membrane protein of Epstein-Barr virus reveal heterogeneity of the protein and inducible expression in virus-transformed cells. J Gen Virol 68: 1575-1586, 1987.

49. Shirakata M, Imadome KI, Okazaki K and Hirai K: Activation of TRAF5 and TRAF6 signal cascades negatively regulates the latent replication origin of Epstein-Barr virus through p38 mitogen-activated protein kinase. J Virol 75: 5059-5068, 2001.
50. Chen C, Li D and Guo N: Regulation of cellular and viral protein expression by the Epstein-Barr virus transcriptional regulator Zta: Implications for therapy of EBV associated tumors. Cancer Biol Ther 8: 987-995, 2009.

51. Tsao SW, Tsang CM, To KF and Lo KW: The role of Epstein-Barr virus in epithelial malignancies. J Pathol 235: 323-333, 2015.

52. Prince S, Keating S, Fielding C, Brennan P, Floettmann E and Rowe M: Latent membrane protein 1 inhibits Epstein-Barr virus lytic cycle induction and progress via different mechanisms. J Virol 77: 5000-5007, 2003.

53. Bentz GL, Moss CR II, Whitehurst CB, Moody CA and Pagano JS: LMP1-induced sumoylation influences the maintenance of Epstein-Barr virus latency through KAP1. J Virol 89: 7465-7477, 2015

54. Lai KY, Chou YC, Lin JH, Liu Y, Lin KM, Doong SL, Chen MR, Yeh TH, Lin SJ and Tsai CH: Maintenance of Epstein-Barr virus latent status by a novel mechanism, latent membrane protein 1-induced interleukin-32, via the protein kinase $\mathrm{C} \delta$ pathway. J Virol 89: 5968-5980, 2015.

55. Adler B, Schaadt E, Kempkes B, Zimber-Strobl U, Baier B and Bornkamm GW: Control of Epstein-Barr virus reactivation by activated CD40 and viral latent membrane protein 1. Proc Natl Acad Sci USA 99: 437-442, 2002.

56. Kim JW, Amin AR and Shin DM: Chemoprevention of head and neck cancer with green tea polyphenols. Cancer Prev Res (Phila) 3: 900-909, 2010.

57. Choi KC, Jung MG, Lee YH, Yoon JC, Kwon SH, Kang HB, Kim MJ,ChaJH,Kim YJ,JunWJ,etal:Epigallocatechin-3-gallate, a histone acetyltransferase inhibitor, inhibits EBV-induced B lymphocyte transformation via suppression of RelA acetylation. Cancer Res 69: 583-592, 2009.

58. Taniguchi S, Imayoshi Y, Kobayashi E, Takamatsu Y, Ito H, Hatano T, Sakagami H, Tokuda H, Nishino H, Sugita D, et al: Production of bioactive triterpenes by Eriobotrya japonica calli. Phytochemistry 59: 315-323, 2002.

59. Chang LK, Wei TT, Chiu YF, Tung CP, Chuang JY, Hung SK, Li C and Liu ST: Inhibition of Epstein-Barr virus lytic cycle by (-)-epigallocatechin gallate. Biochem Biophys Res Commun 301: 1062-1068, 2003. 